\title{
Nonlinear phenomena in nanomechanical resonators: mechanical behaviors and physical limitations
}

\author{
Najib Kacem ${ }^{1,2, a}$, Sébastien Baguet ${ }^{2}$, Sébastien Hentz ${ }^{1}$ and Régis Dufour ${ }^{2}$ \\ 1 CEA/LETI, MINATEC, 17 rue des Martyrs, 38054 Grenoble, France \\ 2 Université de Lyon, CNRS, INSA-Lyon, LaMCoS UMR 5259, 69621 Villeurbanne, France
}

Received 15 February 2010, Accepted 30 November 2010

\begin{abstract}
In order to overcome the loss of performances issue when scaling resonant sensors down to NEMS, it proves extremely useful to study the behavior of resonators up to large displacements and hence high nonlinearities. A comprehensive nonlinear multiphysics model based on the Euler-Bernoulli equation which includes both mechanical and electrostatic nonlinearities in the case of a capacitive doubly clamped beam is presented. This purely analytical model captures all the nonlinear phenomena present in NEMS resonators electrostatically actuated including bistability, multistability which can lead to several physical limitations such as noise mixing, frequency stability deterioration as well as dynamic pull-in. Moreover, close-form expressions of the critical amplitudes and pull-in domain initiation amplitude are provided which can potentially serve for NEMS designers as quick design rules.
\end{abstract}

Key words: NEMS resonator / nonlinear dynamics / critical amplitude / pull-in / mixed behavior

Résumé - Phénomènes non-linéaires dans les résonateurs nanomécaniques : comportements mécaniques et limitations physiques. Afin de compenser la détérioration des performances des capteurs résonants lors de la réduction de leurs tailles au niveau NEMS, il s'avère extrêmement utile d'étudier le comportement des résonateurs en grands déplacements et par conséquent sous fortes non-linéarités. Le modèle de poutre présenté est non-linéaire et multiphysique. Ce capteur capacitif est basé sur l'équation d'Euler-Bernoulli et contient des non-linéarités mécaniques et électrostatiques. Ce modèle, purement analytique, capture tous les phénomènes non-linéaires principaux présents dans des nanorésonateurs capacitifs comme la bistabilité et la multistabilité qui peuvent mener à plusieurs limitations physiques telles que le «noise-mixing », la détérioration de la stabilité en fréquence aussi bien que le «pull-in ». De plus, des expressions analytiques des amplitudes critiques et de l'amplitude d'initiation du domaine «pull-in » sont obtenues, ce qui peut potentiellement servir aux concepteurs comme guides de pré-dimensionnement analytique de NEMS.

Mots clés : Résonateur NEMS / dynamique non-linéaire / amplitude critique / pull-in / comportement mixte

\section{Introduction}

Micro and nanoelectromechanical (MEMS/NEMS) devices have been the subject of extensive research for a number of years and have generated much excitement as their use in commercial applications has increased. Indeed, MEMS technology has opened up a wide variety of potential applications not only in the inertial measurement sector, but also spanning areas such as communications (filters, relays, oscillators, LC passives, optical switches), biomedicine (point-of-care medical

\footnotetext{
${ }^{a}$ Corresponding author: najib.kacem@insa-lyon.fr
}

instrumentation, microarrays for DNA detection and high throughput screening of drug targets, immunoassays, invitro characterization of molecular interactions), computer peripherals (memory, new I/O interfaces, read-write heads for magnetic disks) and other miscellaneous areas such as in projection displays, gas detection and mass flow detection.

NEMS are the natural successor to MEMS as the size of the devices is scaled down to the submicron domain. This transition is well adapted with the resonant sensing technique for a large panel of applications. One reason of scaling down resonant sensors to the NEMS size is the ability to detect very small physical quantities 
by increasing their sensitivity [1]. In particular, NEMS have been proposed for use in ultrasensitive mass detection $[2,3]$, radio frequency $(\mathrm{RF})$ signal processing $[4,5]$, and as a model system for exploring quantum phenomena in macroscopic systems $[6,7]$.

Unfortunately, the nonlinear regime for nanomechanical resonators is easily reachable [8-10], so that the useful linear dynamic range of the smallest NEMS devices is severely limited. In fact, many applications we are hoping for in the near future will involve operation in the nonlinear regime, where the response to the stimulus is suppressed and frequency is pulled away from the original resonant frequency.

The challenge is to achieve large-amplitude motion of NEMS resonators without deteriorating their frequency stability [11]. Indeed, driving the resonator at large oscillation amplitude leads to better signal to noise ratio (SNR) and, thus, simplifies the design of the electronic feedback loop. However, doing so in the nonlinear regime reduces the sensor performances since the frequency instability of a nonlinear resonator is proportional to its oscillation amplitude. Moreover, even when NEMS resonators are used as oscillators in closed-loop, the combination of the mechanical and electrostatic nonlinearities causes a direct voltage-to-frequency effect at the electrodes, leading to so-called noise mixing $[12,13]$ which drastically reduces their dynamic range and therefore makes the detection of resonant peaks more difficult.

The main contribution of this paper consists in explaining in detail the nonlinear phenomena that occur in such a M/NEMS resonator. Section 2 recalls the resonator model including all main sources of nonlinearities and already presented in a previous article [8]. Section 3 is devoted to the description of the different potential nonlinear behaviors due to the competition of mechanical and electrostatic effects. Finally, in Section 4, some inherent nonlinear phenomena which limit the performances of resonant sensors are also highlighted, based on previous work [8].

\section{Model of a nonlinear two-port resonator}

A clamped-clamped beam is considered (Fig. 1) subject to a viscous damping with coefficient $\tilde{c}$ per unit length which models all dissipation sources including ohmic losses $[14,15]$. The device is actuated by an electric load $v(t)=-V_{\mathrm{dc}}+V_{\mathrm{ac}} \cos (\tilde{\Omega} \tilde{t})$, where $V_{\mathrm{dc}}$ is the DC polarization voltage, $V_{\text {ac }}$ the amplitude of the applied AC voltage, and $\tilde{\Omega}$ the excitation frequency.

\subsection{Equation of motion}

Let $E$ be the Young's modulus, $I$, the geometrical moment of inertia of the cross section, $\rho$, the mass density, $l$, the length, $b$, the width, $h$, the thickness of the resonator. Its out-of-plane deflection $\tilde{w}(x, t)$ which depends on $\tilde{c}$, the viscous damping, $\tilde{N}$ the axial load, and on the forces provided by the two electrodes is governed by the following nonlinear Euler-Bernoulli equation which is the commonly used approximate equation of motion for a thin beam [16].

$$
\begin{gathered}
\rho b h \frac{\partial^{2} \tilde{w}(\tilde{x}, \tilde{t})}{\partial \tilde{t}^{2}}+\tilde{c} \frac{\partial \tilde{w}(\tilde{x}, \tilde{t})}{\partial \tilde{t}}+E I \frac{\partial^{4} \tilde{w}(\tilde{x}, \tilde{t})}{\partial \tilde{x}^{4}} \\
-\left[\tilde{N}+\frac{E b h}{2 l} \int_{0}^{l}\left[\frac{\partial \tilde{w}(\tilde{x}, \tilde{t})}{\partial \tilde{x}}\right]^{2} \mathrm{~d} \tilde{x}\right] \frac{\partial^{2} \tilde{w}(\tilde{x}, \tilde{t})}{\partial \tilde{x}^{2}} \\
=\frac{1}{2} \varepsilon_{0} \frac{b C_{n 1}\left[V_{\mathrm{ac}} \cos (\tilde{\Omega} \tilde{t})-V_{\mathrm{dc}}\right]^{2}}{\left(g_{\mathrm{a}}-\tilde{w}(\tilde{x}, \tilde{t})\right)^{2}} H_{1}(\tilde{x}) \\
-\frac{1}{2} \varepsilon_{0} \frac{b C_{n 2}\left[V_{\mathrm{s}}-V_{\mathrm{dc}}\right]^{2}}{\left(g_{\mathrm{d}}+\tilde{w}(\tilde{x}, \tilde{t})\right)^{2}} H_{2}(\tilde{x}) \\
H_{1}(\tilde{x})=H\left(\tilde{x}-\frac{l+l_{\mathrm{a}}}{2}\right)-H\left(\tilde{x}-\frac{l-l_{\mathrm{a}}}{2}\right) \\
H_{2}(\tilde{x})=H\left(\tilde{x}-\frac{l+l_{\mathrm{d}}}{2}\right)-H\left(\tilde{x}-\frac{l-l_{\mathrm{d}}}{2}\right)
\end{gathered}
$$

where $\varepsilon_{0}$ is the dielectric constant of the gap medium. The two terms of the right-hand member in Equation (1) represent an approximation of the electric forces assuming a resonator design with two stationary electrodes: electrode 1 for the actuation and electrode 2 for the sensing including the fringing field effect [17] using the coefficients $C_{n i}$. The boundary conditions are:

$$
\tilde{w}(0, \tilde{t})=\tilde{w}(l, \tilde{t})=\frac{\partial \tilde{w}}{\partial \tilde{x}}(0, \tilde{t})=\frac{\partial \tilde{w}}{\partial \tilde{x}}(l, \tilde{t})=0
$$

\subsection{Nondimensionalisation}

For convenience, let the following nondimensional variables be introduced:

$$
w=\frac{\tilde{w}}{g_{\mathrm{d}}}, x=\frac{\tilde{x}}{l}, \quad t=\frac{\tilde{t}}{\tau}
$$

where $\tau=\frac{2 l^{2}}{h} \sqrt{\frac{3 \rho}{E}}$. Substituting Equation (5) into Equations (1)-(4), gives:

$$
\begin{gathered}
\frac{\partial^{2} w}{\partial t^{2}}+c \frac{\partial w}{\partial t}+\frac{\partial^{4} w}{\partial x^{4}}-\left[N+\alpha_{1} \int_{0}^{1}\left[\frac{\partial w}{\partial x}\right]^{2} \mathrm{~d} x\right] \\
\times \frac{\partial^{2} w}{\partial x^{2}}+\alpha_{2} C_{n 2} \frac{\left[V_{\mathrm{s}}-V_{\mathrm{dc}}\right]^{2}}{(1+w)^{2}} H_{2}(x)= \\
\alpha_{2} C_{n 1} \frac{\left[V_{\mathrm{ac}} \cos (\Omega t)-V_{\mathrm{dc}}\right]^{2}}{\left(R_{\mathrm{g}}-w\right)^{2}} H_{1}(x) \\
w(0, t)=w(1, t)=\frac{\partial w}{\partial x}(0, t)=\frac{\partial w}{\partial x}(1, t)=0
\end{gathered}
$$




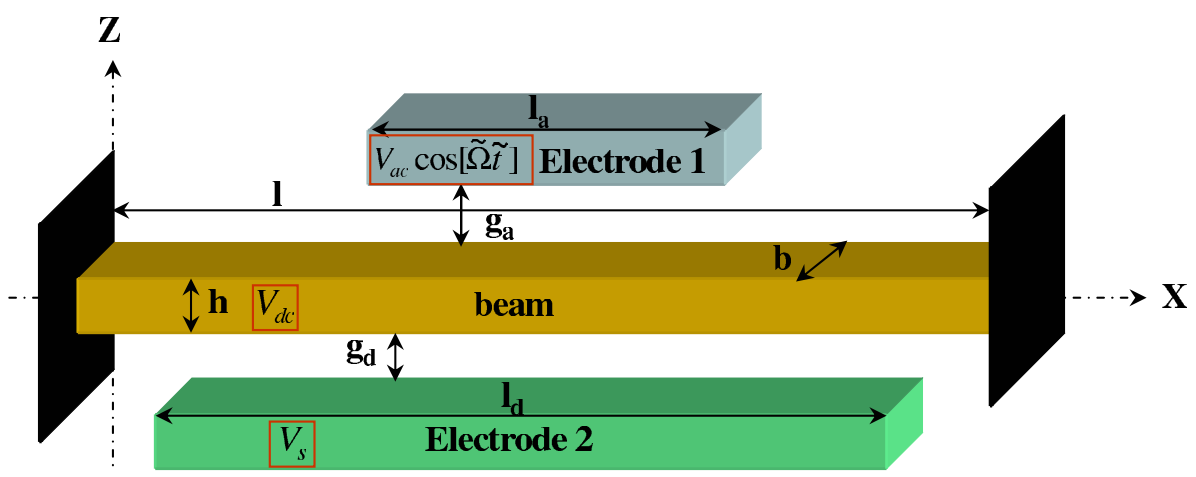

Fig. 1. Two-ports resonator layout.

The parameters appearing in Equation (6) are:

$$
\begin{aligned}
H_{2}(x) & =H\left(x-\frac{l+l_{\mathrm{a}}}{2 l}\right)-H\left(x-\frac{l-l_{\mathrm{a}}}{2 l}\right) \\
H_{2}(x) & =H\left(x-\frac{l+l_{\mathrm{d}}}{2 l}\right)-H\left(x-\frac{l-l_{\mathrm{d}}}{2 l}\right) \\
c & =\frac{\tilde{c} l^{4}}{E I \tau}, \quad N=\frac{\tilde{N} l^{2}}{E I}, \quad \alpha_{1}=6\left[\frac{g_{\mathrm{a}}}{h}\right]^{2} \\
R_{\mathrm{g}} & =\frac{g_{\mathrm{a}}}{g_{\mathrm{d}}}, \quad \alpha_{2}=6 \frac{\varepsilon_{0} l^{4}}{E h^{3} g_{\mathrm{a}}^{3}}, \quad \Omega=\tilde{\Omega} \tau
\end{aligned}
$$

\subsection{Reduced order model}

A reduced-order model is generated by modal decomposition transforming Equation (6) into a discrete system consisting of ordinary differential equations in time. For this end, the Galerkin procedure is used and the deflection is expanded on the undamped linear mode shapes of the straight beam:

$$
w(x, t)=\sum_{k=1}^{n} a_{k}(t) \phi_{k}(x)
$$

where $n$ is the number of retained modes, $a_{k}(t)$ is the $k$ th generalized coordinate and $\phi_{k}(x)$ is the $k$ th linear undamped mode shape of the straight non-prestressed beam, normalized such that $\int_{0}^{1} \phi_{k} \phi_{j}=\delta_{k j}$ where $\delta_{k j}=0$ if $k \neq j$ and $\delta_{k j}=1$ if $k=j$. The linear undamped mode shapes $\phi_{k}(x)$ are governed by:

$$
\begin{array}{r}
\frac{\mathrm{d}^{4} \phi_{\mathrm{k}}(x)}{\mathrm{d} x^{4}}=\lambda_{k}^{2} \phi_{k}(x) \\
\phi_{k}(0)=\phi_{k}(1)=\phi_{k}^{\prime}(0)=\phi_{k}^{\prime}(1)
\end{array}
$$

Let Equation (6) be multiplied by $\phi_{k}(x)\left[(1+w)\left(R_{g}-w\right)\right]^{2}$ in order to include the complete contribution of the nonlinear electrostatic forces in the resonator dynamics without approximation. This method, which has been used by Nayfeh et al. [18] for a one-port resonator, has some disadvantages like the non orthogonality of the operator $w^{4} \frac{\partial^{4} w}{\partial x^{4}}$ with respect to the undamped linear mode shapes of the resonator, the increase of the nonlinearity level in the normalized equation of motion (6) as well as the incorporation of new nonlinear terms such as the Van der Pol damping. Nevertheless, the resulting equation contains less parametric terms than if the nonlinear electrostatic forces were expanded in Taylor series and the solution of nonlinear problem is valid for large displacements of the beam up to the sensing gap.

Next, we substitute Equation (12) into the resulting equation, use Equation (13) to eliminate $\frac{\mathrm{d}^{4} \phi_{k}(x)}{\mathrm{d} x^{4}}$, integrate the outcome from $x=0$ to 1 , and obtain a system of coupled ordinary differential equations in time.

For several nonlinear mechanical systems such as shells [19], many modes are needed in order to build the frequency response without losing any physical information concerning the nonlinear coupling between the modes.

Nevertheless, in our case (simple beam resonators), the first mode can be considered as the dominant mode of the system and the higher modes could be neglected which has been demonstrated numerically using time integration [8] as well as shooting and harmonic balance method coupled with an asymptotic numerical continuation technique [20]. Thus, only one mode is considered $(n=1)$ and the following equation is obtained:

$$
\begin{gathered}
\ddot{a}_{1}+c \dot{a}_{1}+\omega_{1}{ }^{2} a_{1}+\mu_{1} a_{1} \ddot{a}_{1}+\mu_{2} a_{1}{ }^{2} \ddot{a}_{1}+\mu_{3} a_{1}{ }^{3} \ddot{a}_{1}+\mu_{4} a_{1}{ }^{4} \\
+c \mu_{3} a_{1}{ }^{3} \dot{a}_{1}+c \mu_{4} a_{1}{ }^{4} \dot{a}_{1} \ddot{a}_{1}+c \mu_{1} a_{1} \dot{a}_{1}+c \mu_{2} a_{1}{ }^{2} \dot{a}_{1} \\
+\chi_{2} a_{1}{ }^{2}+\chi_{3} a_{1}{ }^{3}+\chi_{4} a_{1}{ }^{4}+\chi_{5} a_{1}{ }^{5}+\chi_{6} a_{1}{ }^{6}+\chi_{7} a_{1}{ }^{7}+\nu \\
+\zeta_{0} \cos (\Omega t)+\zeta_{1} a_{1} \cos (\Omega t)+\zeta_{2} a_{1}{ }^{2} \cos (\Omega t)+\zeta_{3} \cos (2 \Omega t) \\
+\zeta_{4} a_{1} \cos (2 \Omega t)+\zeta_{5} a_{1}{ }^{2} \cos (2 \Omega t)=0
\end{gathered}
$$

where $\omega_{1}$ is the natural frequency of the first mode and $\nu$ is the static force. $\chi_{i \in\{2,3,4,5,6,7\}}$ are the nonlinear stiffnesses and $\zeta_{i \in\{0,3\}}$ are the amplitudes of the time varying linear forces. $\mu_{i \in\{1,2,3,4\}}$ and $c \mu_{i \in\{1,2,3,4\}}$ are the nonlinear acceleration and damping terms which come from the multiplication of Equation (6) by the common denominator of the electrostatic forces and $\zeta_{i \in\{1,2,4,5\}}$ are the amplitudes of the parametric forces coming from the coupling between the actuation and sensing electrodes.

Equation (15) contains canonical nonlinear terms such as nonlinear stiffness $\left(\chi_{3} a_{1}^{3}\right)$, nonlinear damping $\left(c \mu_{2} a_{1}^{2} a_{1}^{\prime}\right)$ as well as parametric excitation 
$\left(\zeta_{1} a_{1} \cos (\Omega t)\right)$. Therefore, Equation (15) governs the behavior of a Duffing-Van der Pol-Mathieu oscillator. Moreover, the presence of other high-level nonlinearities in Equation (15) makes the described system as a forced nonlinear resonator under multifrequency parametric excitation. For a two-port resonator, this kind of equation is similar to the one used by Nayfeh et al. [18] for a one-port resonator and includes terms coming from the coupling between the mechanical and the electrostatic nonlinearities as well as the nonlinear coupling between both electrostatic forces. The expressions of all the integration parameters presented in Equation (15) are listed in [8]. They can be easily computed with any computational software.

To analyze this equation of motion, we use perturbation techniques which are well adapted to "small" excitation and damping $(Q>10)$, typically valid in NEMS resonators [21]. Since the device operates near resonance, a detuning parameter is introduced as $\Omega=$ $\omega_{n}+\varepsilon \sigma$ and the averaging method [22] is used in order to transform Equation (15) into two first order nonlinear ordinary-differential equations that modulate the amplitude $A$ and the phase $\beta$ of the transformation $a_{1}=$ $A(t) \cos [\Omega t+\beta(t)]$ :

$$
\begin{aligned}
\dot{A} & =\varepsilon \frac{\zeta_{0} \sin \beta}{2 \omega_{n}}+\varepsilon \frac{A^{2} \zeta_{2} \sin \beta}{8 \omega_{n}}-\varepsilon \frac{A c}{2} \\
& \quad-\varepsilon \frac{A^{3} c \mu_{2}}{8}-\varepsilon \frac{A^{5} c \mu_{4}}{16}+O\left(\varepsilon^{2}\right) \\
\dot{\beta} & =\varepsilon \sigma-\varepsilon \frac{3 A^{2} \chi_{3}}{8 \omega_{n}}-\varepsilon \frac{5 A^{4} \chi_{5}}{16 \omega_{n}}-\varepsilon \frac{35 A^{6} \chi_{7}}{128 \omega_{n}}-\varepsilon \frac{\zeta_{0} \cos \beta}{2 A \omega_{n}} \\
& -\varepsilon \frac{3 A \zeta_{2} \cos \beta}{8 \omega_{n}}+\varepsilon \frac{3}{8} A^{2} \omega_{n} \mu_{2}+\varepsilon \frac{5}{16} A^{4} \omega_{n} \mu_{4}+O\left(\varepsilon^{2}\right)
\end{aligned}
$$

The steady-state motions occur when $\dot{A}=\dot{\beta}=0$, which corresponds to the singular points of Equations (16) and (17). Thus, the frequency-response equation can be written in its parametric form $\left\{A=K_{1}(\beta), \Omega=K_{2}(\beta)\right\}$ as a function of the phase $\beta$ as a set of 2 equations easy to introduce in Matlab or Mathematica. This ability makes the model suitable for NEMS designers as a quick tool of resonant sensor performance optimization. For the sake of clarity, the frequency-response equation can be written in its implicit form as:

$$
\begin{gathered}
\frac{A^{2}\left\{\Lambda_{1}(A)-8 \omega_{n}\left(16 \Omega+\Lambda_{2}(A)\right)\right\}^{2}}{256\left(4 \zeta_{0}+3 A^{2} \zeta_{2}\right)^{2}} \\
+\frac{\left(8 c A+2 A^{3} c \mu_{2}+A^{5} c \mu_{4}\right)^{2} \omega_{n}^{2}}{4\left(4 \zeta_{0}+A^{2} \zeta_{2}\right)^{2}}=1 \\
\Lambda_{1}(A)=48 A^{2} \chi_{3}+40 A^{4} \chi_{5}+35 A^{6} \chi_{7} \\
\Lambda_{2}(A)=\left(6 A^{2} \mu_{2}+5 A^{4} \mu_{4}-16\right) \omega_{n}
\end{gathered}
$$

\section{Typical behaviors}

All the numerical simulations were carried out for a device with the following parameters: $l=20 \mu \mathrm{m}$, $b=4 \mu \mathrm{m}, h=2 \mu \mathrm{m}, l_{\mathrm{a}}=20 \mu \mathrm{m}, l_{\mathrm{d}}=112 \mu \mathrm{m}$ and $\mathrm{an}$

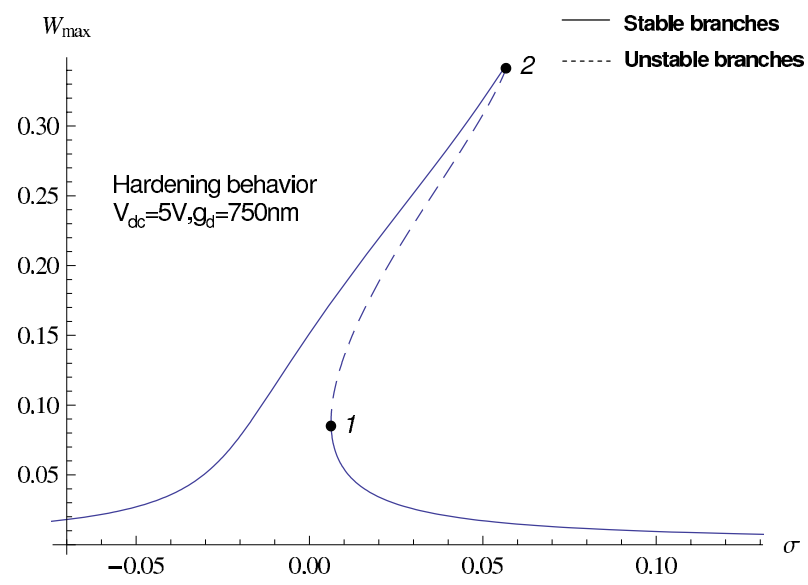

Fig. 2. Hardening behavior of the typical resonator described in Figure 1. $\sigma$ is the detuning parameter, $W_{\max }$ is the displacement of the beam normalized by the gap $g_{\mathrm{d}}$ at its middle point $\frac{l}{2}$ and $\{1,2\}$ are the bifurcation points.

axial residual stress on the beam material $\sigma_{\mathrm{r}}=15 \mathrm{MPa}$. $V_{\mathrm{s}}$ is set to zero volt which has no impact on the generality of the analytical investigations since the electrostatic force is proportional to the square of the electric potential difference. Both gaps can be used to tune the electrostatic nonlinear effect. Nevertheless, for convenience, the gap between the beam and the actuation electrode $g_{\mathrm{a}}$ is set to $1 \mu \mathrm{m}$. $V_{\mathrm{ac}}, V_{\mathrm{dc}}$ and $g_{\mathrm{d}}$ were used for parametric analysis.

These dimensions are extracted from the design of a resonant accelerometer which has been manufactured in CEA LETI clean rooms in order to investigate the nonlinear dynamics of resonant sensors within the framework of the transition from MEMS to NEMS [8].

\subsection{Hardening behavior}

At the micro and nanoscale, the spring hardening is the most classical effect observed in clamped-clamped resonators electrostatically actuated $[23,24]$. The mechanical nonlinearities due to mid-plane stretching dominate the resonator dynamics and the frequency response peak is hysteretic and shifted to the high frequencies (Fig. 2). Furthermore, when the mechanical nonlinearities are preponderant, the dynamics of one and two ports resonators are equivalent.

\subsection{Softening behavior}

In order to increase the softening electrostatic nonlinearities, the resonator designs have to involve very narrow gaps with respect to the beam width which is of great difficulty with Top-Down technology for NEMS. Therefore the softening behavior is difficult to obtain in clampedclamped NEMS resonators electrostatically actuated. For the considered resonator, the softening behavior is possible for a sensing gap $g_{\mathrm{d}}=400 \mathrm{~nm}$ as shown in Figure 3 . The frequency response curve is hysteretic and shifted to the low frequencies. 


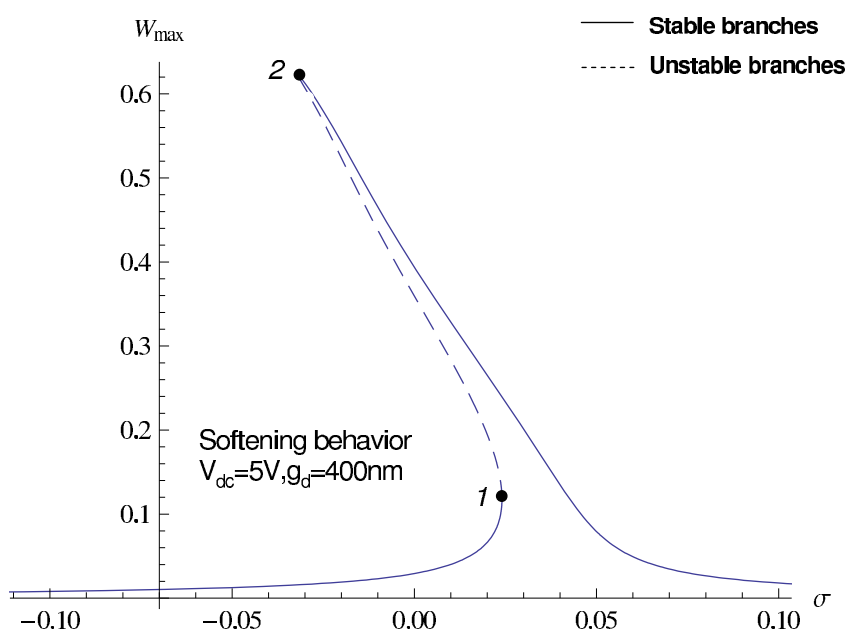

Fig. 3. Softening behavior of the typical resonator described in Figure 1. $\sigma$ is the detuning parameter, $W_{\max }$ is the displacement of the beam normalized by the gap $g_{\mathrm{d}}$ at its middle point $\frac{l}{2}$ and $\{1,2\}$ are the bifurcation points.

\subsection{Mixed behavior}

Both mechanical and electrostatic nonlinearities are always operating into the system. However, in some configurations one kind of nonlinearity is negligible with respect to the second one. Practically, when $h / g_{\mathrm{d}} \ll 1$, then the dynamics is dominated by the hardening nonlinearities and in the opposite case $\left(h / g_{\mathrm{d}} \gg 1\right)$, the frequency response is nonlinearly softening. Between these two configurations and for the typical manufactured resonator described in Figure 1, the hardening behavior could occur for low DC voltages. Then, when $V_{\mathrm{dc}}$ is increased up to $8 \mathrm{~V}$, the nonlinear softening stiffness coming from the electrostatic becomes sufficiently large to create a mixed hardening-softening behavior described in Figure 4 except for an optimal gap $g_{\mathrm{d}}=500 \mathrm{~nm}$ for which both nonlinearities could be perfectly equilibrated [8]. The mixed behavior is characterized by a four-bifurcation points frequency response and up to 5 amplitude for a given frequency [25]. In the present case of Figure 4, the bifurcation point 3 can not be reached since the $\mathrm{P}$ point is at a higher frequency than the bifurcation point 1 and thus, at this point, the jump up in sweep down frequency brings the solution at the point $d_{3}$ of the lower branch where the basin of attractions is quite large. Hence, the upper branch between $d_{1}$ and 3 is unstable. Indeed, the mixed behavior is highly unstable, dangerous and thus undesirable for MEMS and NEMS designers.

\section{Physical limitations due to nonlinear phenomena}

\subsection{Critical amplitude}

The critical amplitude is the oscillation amplitude $A_{\mathrm{c}}$ above which bistability occurs. Thus, $A_{\mathrm{c}}$ is the transition

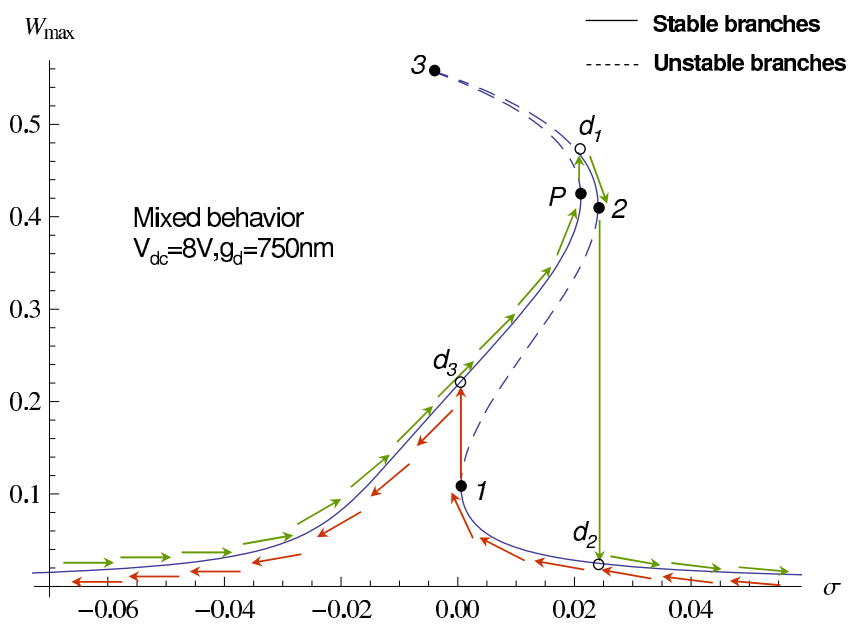

Fig. 4. Mixed behavior of the typical resonator described in Figure 1 for $g_{\mathrm{d}}=750 \mathrm{~nm}, V_{\mathrm{dc}}=8 \mathrm{~V}$ and $V_{\mathrm{ac}}=10^{-2} V_{\mathrm{dc}} \cdot \sigma$ is the detuning parameter, $W_{\max }$ is the displacement of the beam normalized by the gap $g_{\mathrm{d}}$ at its middle point $\frac{l}{2}$ and $\{1,2,3, P\}$ are the different bifurcation points.

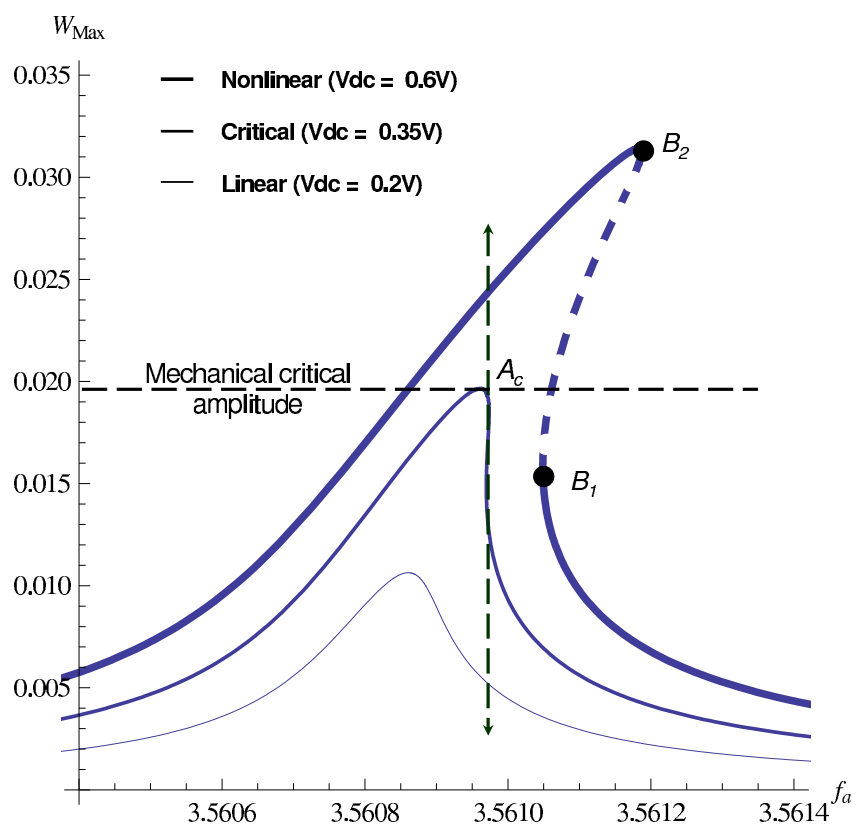

Fig. 5. Forced frequency responses of the resonator. $f_{\mathrm{a}}$ is the dimensionless frequency and $W_{\max }$ is the displacement of the beam normalized by the gap $g_{\mathrm{d}}$ at its middle point $\frac{l}{2}$. $A_{\mathrm{c}}$ is the mechanical critical amplitude and $\left\{B_{1}, B_{2}\right\}$ are the two bifurcation points of a typical hardening behavior.

amplitude from the linear to the nonlinear behavior (see Fig. 5). At the critical drive, the resonance curve exhibits a point of infinite slope, called the critical point. Moreover, at the same point, the phase curve also exhibits an infinite slope at the same detuning as the resonance curve itself. Nayfeh studied the stability of an excited Duffing oscillator [22]. Kaajakari [26] provided close form expression for the critical amplitude using a reduced order model including the crystalline direction of beam resonators. However, the complete contribution of the electrostatic 
nonlinearities was not incorporated. In other words, he simply provided the mechanical critical amplitude.

\subsubsection{Mechanical critical amplitude}

For the primary resonance of a clamped-clamped beam, $A_{\mathrm{c}}$ is defined as the oscillation amplitude for which the equation $\frac{\mathrm{d} \sigma}{\mathrm{d} \beta}=0$ has a unique solution. In order to explain how to deduce the mechanical critical amplitude from Equation (18) written in its parametric form, let the nonlinear electrostatic effects be neglected $\left(h / g_{\mathrm{d}} \ll 1\right)$. The parametric form of the frequency response can be written as:

$$
\begin{aligned}
\sigma & =\frac{1}{8}\left(\frac{3 \zeta_{0}^{2} \chi_{3}}{c^{2} \omega_{1}^{3}} \sin ^{2} \beta-4 c \cot \beta\right) \\
A & =\frac{\zeta_{0}}{c \omega_{n}} \sin \beta
\end{aligned}
$$

Using Equation (21) permits the differentiation of the detuning parameter $\sigma$ with respect to the phase $\beta$ :

$$
\frac{\mathrm{d} \sigma}{\mathrm{d} \beta}=\frac{6 \chi_{3} \zeta_{0}^{2} \sin 2 \beta-3 \chi_{3} \zeta_{0}^{2} \sin 4 \beta+16 c^{3} \omega_{1}^{3}}{32 c^{2} \omega_{1}^{3} \sin ^{2} \beta}
$$

One can search the condition for which the equation $6 \chi_{3} \zeta_{0}^{2} \sin 2 \beta-3 \chi_{3} \zeta_{0}^{2} \sin 4 \beta+16 c^{3} \omega_{1}^{3}=0$ has solutions. This equation can be written as $\Delta(2 \sin 2 \beta-\sin 4 \beta)+1=0$ where $\Delta=\frac{3 \zeta_{0}^{2} \chi_{3}}{16 c^{3} \omega_{1}^{3}}$ and then, transformed to a fourth order polynomial equation by using the change of variable $X=\sin 2 \beta$.

$$
1+4 \Delta X+4 \Delta^{2} X^{4}=0
$$

Equation (24) can be solved using the Ferrari's method and the following cases are obtained:

$$
\left\{\begin{array}{c}
\text { If } \Delta<\frac{2}{3 \sqrt{3}} \Longrightarrow \text { No solutions Linear behavior } \\
\text { If } \Delta=\frac{2}{3 \sqrt{3}} \Longrightarrow \text { A unique solution } \beta_{c}=\frac{2 \pi}{3} \\
\text { Critical behavior } \\
\text { If } \Delta>\frac{2}{3 \sqrt{3}} \Longrightarrow 2 \text { distinct solutions } \beta_{1} \text { et } \beta_{2} \\
\text { Nonlinear behavior }
\end{array}\right.
$$

The condition $\Delta=\frac{2}{3 \sqrt{3}}$ implies the following relation

$$
\zeta_{0 c}=\frac{4 \sqrt{2} c^{3 / 2} \omega_{1}^{3 / 2}}{3 \sqrt[4]{3} \sqrt{\chi_{3}}}
$$

$\zeta_{0 c}$ corresponds to the critical drive for which the resonance curve exhibits an infinite slop at the phase $\beta_{\mathrm{c}}=\frac{2 \pi}{3}$ and the two bifurcation points $B_{1}$ and $B_{2}$ are superposed. When this condition is satisfied, the mechanical critical amplitude is computed at the resonance peak. It is the value of Equation (22) at the phase $\beta=\frac{\pi}{2}$ (for a critical behavior) multiplied by 1.588 which correspond to the amplification coefficient of the first mode at the middle of the clamped-clamped beam $x=\frac{1}{2}$. Substituting Equation (25) in the resulting equation and mutiplying by the gap $g_{\mathrm{d}}$ to obtain the dimensional value, yields:

$$
A_{\mathrm{c}_{\mathrm{m}}}=\frac{2.275 g_{\mathrm{d}} \sqrt{c} \sqrt{\omega_{1}}}{\sqrt{\chi_{3}}}
$$

Substituting $\omega_{1}, c$ and $\chi_{3}$ expressions in Equation (26), yields:

$$
A_{\mathrm{c}_{\mathrm{m}}}=1.685 \frac{h}{\sqrt{Q}}
$$

where $Q=\rho b h \frac{\omega_{1}}{\tau \tilde{c}}$ is the quality factor of a resonant clamped-clamped beam under its first bending vibration mode. By using the reduced order model and the first order averaging method, the mechanical critical amplitude was easily deduced and the obtained close-form solution is comparable to those defined in $[24,26]$.

\subsubsection{Global critical amplitude}

The close-form expression of the mechanical critical amplitude $A_{\mathrm{c}_{\mathrm{m}}}$ has been provided using the nonlinear model of a clamped-clamped resonator while neglecting the effects of the electrostatic nonlinearities. Indeed, Equation (27) represents the classical form of the critical amplitude for a Duffing resonator (only mechanical nonlinearity) [27]. It shows that the critical amplitude is only determined by the beam thickness in the direction of vibration $h$ and the quality factor $Q$ and does not depend on the beam length $l$. This information has been observed experimentally by Shao et al. [24] for micromechanical clamped-clamped beam resonators using stroboscopic SEM. Our model allows the computation of the global critical amplitude when all sources of nonlinearities are included: it can be deduced using the same method as explained in the simplified case for the mechanical critical amplitude.

$$
\begin{aligned}
A_{c} & =\sqrt{\theta_{1} h^{2}-\theta_{2}+\sqrt{\theta_{3}-\theta_{4} h^{2}+\theta_{5} h^{4}+\frac{\theta_{6} h^{2}}{Q}}} \\
\theta_{1} & =3.6327 \times 10^{-3} \\
\theta_{2} & =\frac{19328 g_{\mathrm{d}}^{2}}{43375} \\
\theta_{3} & =0.19856 g_{\mathrm{d}}^{4} \\
\theta_{4} & =3.2375 \times 10^{-3} g_{\mathrm{d}}^{2} \\
\theta_{5} & =1.3197 \times 10^{-5} \\
\theta_{6} & =2.5683 g_{\mathrm{d}}^{2}
\end{aligned}
$$

Remarkably the mechanical critical amplitude (Eq. (27)) depends only on the thickness of the beam $h$ and the quality factor $Q$. However, in addition to these two parameters, the global critical amplitude (Eqs. (28)-(34)) depends also on the detection gap $g_{\mathrm{d}}$.

It can easily checked that $\lim _{g_{\mathrm{d}} \rightarrow \infty} A_{\mathrm{c}}=A_{\mathrm{c}_{\mathrm{m}}}$. 
For example, the critical amplitude of a resonator having a quality factor of $10^{4}$ designed with $100 \mathrm{~nm}$ of thickness in the direction of vibration and a sensing gap of $200 \mathrm{~nm}$, is about $1.68 \mathrm{~nm}$.

In order to stay linear, the restrictive amplitude is $0.84 \%$ of the sensing gap, which leads to a very weak signal to noise ratio (SNR) and thus a very poor resolution.

\subsection{Pull-in}

As it can be observed in Equation (1), the electrostatic force is inversely proportional to the square of the gap between the beam and the electrode. As the gap decreases, the generated attractive force increases quadratically. The only opposing force to the electrostatic loading is the mechanical restoring force. If the voltage increases, the gap decreases generating an incremented force. At some point the mechanical forces defined by the spring cannot balance this force anymore. Once this state is reached, the beam snaps against the electrode, and in most cases, the system is permanently disabled. Consequently, the electrostatic loading has an upper limit beyond which the mechanical force can no longer resist the opposing electrostatic force, thereby leading to the collapse of the structure. This actuation instability phenomenon is known as pull-in, and the associated critical voltage is called the pull-in voltage.

\subsubsection{Static pull-in}

To derive the expression for pull-in, let us consider a one-port resonator [28]. Neglecting the mechanical nonlinearities, the total potential energy in the system can be written as follows:

$$
E_{\mathrm{p}}=-\frac{1}{2} \frac{\epsilon_{0} b l}{g-u} V_{\mathrm{dc}}^{2}+\frac{1}{2} k u^{2}
$$

where $u$ is the average displacement of the resonator and $g$ is the gap between the beam and the electrode. The first term in Equation (35) is the electrostatic potential of the deformable capacitor (the resonator) and the second term is due to the mechanical energy stored in the spring ( $k$ is the effective spring constant of the resonator). The force acting on the movable beam is obtained by deriving Equation (35):

$$
F=-\frac{\partial E_{\mathrm{p}}}{\partial u}=\frac{1}{2} \frac{\epsilon_{0} b l}{(g-u)^{2}} V_{\mathrm{dc}}^{2}-k u
$$

At equilibrium, the electrostatic force and spring force cancel $(F=0)$ and Equation (36) gives:

$$
k u=\frac{1}{2} \frac{\epsilon_{0} b l}{(g-u)^{2}} V_{\mathrm{dc}}^{2}
$$

Equation (37) can be solved for the equilibrium beam position $\tilde{w}$ as a function of applied voltage $V_{\mathrm{dc}}$. Above the pull-in voltage $V_{\mathrm{P}}$, Equation (37) has no solution. A simple expression for the pull-in point is deduced by deriving Equation (36) to obtain the stiffness of the system:

$$
\frac{\partial F}{\partial u}=\frac{\epsilon_{0} b l}{(g-u)^{3}} V_{\mathrm{dc}}^{2}-k
$$

Substituting Equation (37) gives the stiffness around the equilibrium point:

$$
\frac{\partial F}{\partial u}=\frac{2 k u}{g-u}-k
$$

With no applied voltage Equation (39) is simply $\frac{\partial F}{\partial u}=$ $-k$; a virtual positive deflection $\delta u$ induces a negative restoring force $\frac{\partial F}{\partial u} \delta u=-k \delta u$. Increasing the bias voltage $V_{\mathrm{dc}}$ makes the stiffness less negative. The unstable point is given by $\frac{\partial F}{\partial u}=0$ giving

$$
u=\frac{1}{3} g
$$

Beyond this point the stiffness becomes positive and the system is unstable: a virtual positive deflection $\delta u$ induces a positive force that increases $u$. Substituting Equation (40) to Equation (37) gives the pull-in voltage at which the system becomes unstable

$$
V_{\mathrm{P}}=\sqrt{\frac{8 k g^{3}}{27 \epsilon_{0} b l}}
$$

\subsubsection{Dynamic pull-in}

The pull-in amplitude is the oscillation amplitude above which the resonator position becomes unstable and collapses. The dynamic pull-in is the collapse of the beam subjected to a time-varying electrostatic force. It is completely different from the static pull-in [27] where the electrostatic force depends only on the gap.

In the general case, pull-in can occur for multistable hardening and softening behaviors. Nevertheless, for practical cases of nanoresonators which are designed with gaps and width in the direction of vibration of the same order of magnitude, the mixed behavior occurs before pull-in is reached.

Consequently, and like other studies [29], we define point $\mathrm{P}$ as the initiation of an unstability domain and thus an upper bound of possible drive, beyond which dynamic pull-in is likely to occur. Thus, the pull-in domain initiation amplitude is located at the bifurcation point $\mathrm{P}$ where the effect of the nonlinear electrostatic stiffness becomes significantly important, and where the domain of attraction of stable branches is small, making the jump of the system to these stable branches quite hard physically [18].

Following [8], the initiation of the dynamic pull-in domain can be located via the transition from two to three bifurcation points. The third bifurcation point is situated at the phase $\beta=\frac{\pi}{2}$ which corresponds to the initiation of 
the mixed behavior. The latter is characterized by a slope approaching infinity at point $\mathrm{P}$ as shown in Figure 4 and it has been demonstrated analytically and experimentally that the amplitude of this bifurcation point is set by the geometry of the resonator [25]. Hence, the following expression of the pull-in voltage $V_{\mathrm{p}}$ :

$$
\begin{aligned}
V_{\mathrm{p}} & =\left\|\frac{3 l \pi c \omega_{n} \Delta\left(-\chi_{3}+\mu_{2} \omega_{n}^{2}\right)^{\frac{1}{2}}\left[\chi_{5}-\mu_{4} \omega_{n}^{2}\right]^{-\frac{5}{2}}}{400 \sqrt{10} \alpha_{2} C_{n 1}\left(l^{2} \cos \left[\frac{\pi}{l}\right] \sin \left[\frac{\pi l_{d}}{l^{2}}\right]-\pi l_{d}\right)}\right\| \\
\Delta & =200 \chi_{5}^{2}+\mu_{4}\left(-21 \mu_{2}^{2}+200 \mu_{4}\right) \omega_{n}^{4}-30 \mu_{2} \chi_{3} \chi_{5} \\
& +2\left(6 \mu_{2} \mu_{4} \chi_{3}+5\left(3 \mu_{2}^{2}-40 \mu_{4}\right) \chi_{5}\right) \omega_{n}^{2}+9 \mu_{4} \chi_{3}^{2}
\end{aligned}
$$

\section{Conclusion}

This work has detailed the development of an analytical model to qualitatively and quantitatively assess the nonlinear phenomena of NEMS resonators. This model includes the main sources of nonlinearities, in particular the electrostatic ones, without approximation and is based on the modal decomposition using the Galerkin procedure combined with the averaging perturbation technique method.

It has been shown that the model is able to capture all the nonlinear phenomena in the resonator dynamics including bistability and multistability. Beyond the occurrence of such phenomena, the sensor frequency stability is extremely altered. Combined with the noise mixing issue $[12,13]$, this leads to drastic losses in the resonator performances. The developed analytical model proves to be a practical tool for NEMS designers since it provides close-form expressions of the critical amplitudes as well as the pull-in domain initiation amplitude.

Acknowledgements. The authors are indebted of the Carnot Institutes CEA-LETI and I@L for their supports.

\section{References}

[1] K.L. Ekinci, M.L. Roukes, Nanoelectromechanical systems, Rev. Sci. Instrum. 76 (2005) 061101

[2] X.M.H. Huang, K.L. Ekinci, M.L. Roukes, Ultrasensitive nanoelectromechanical mass detection, Appl. Phys. Lett. 84 (2004) 4469-4471

[3] K. Jensen, K. Kim, A. Zettl, An atomic-resolution nanomechanical mass sensor, Nature Nanotechnol. 3 (2008) 533-537

[4] C.T. Nguyen, Micromechanical components for miniaturized low-power communications, in 1999 IEEE MTT-S international Microwave Symposium FR MEMS Workshop, 1999, pp. $48-77$
[5] A.C.W. Nguyen, C.T.D. Hao, Tunable, switchable, highq vhf microelectromechanical bandpass filters, in IEEE International Solid-State Circuits Conf., 1999, Vol. 448, p. 78

[6] A. Cho, Physics-researchers race to put the quantum into mechanics, Science 299 (2003) 36-37

[7] M.D. LaHaye, O. Buu, B. Camarota, K.C. Schwab, Approaching the quantum limit of a nanomechanical resonator, Science 304 (2004) 74-77

[8] N. Kacem, S. Hentz, D. Pinto, B. Reig, V. Nguyen, Nonlinear dynamics of nanomechanical beam resonators: improving the performance of nems-based sensors, Nanotechnology 20 (2009) 275501

[9] N. Kacem, J. Arcamone, F. Perez-Murano, S. Hentz, Dynamic range enhancement of nonlinear nanomechanical resonant cantilevers for high sensitive NEMS gas/mass sensors applications, J. Micromech. Microeng. 20 (2010) 04502

[10] B.G. Sumpter, D.W. Noid, The onset of instability in nanostructures: the role of nonlinear resonance, J. Chem. Phys. 102 (1995) 6619-6622

[11] X.L. Feng, Phase noise and frequency stability of veryhigh frequency silicon nanowire nanomechanical resonators, in 14th International Conference on Solid-State Sensors, Actuators and Microsystems, 2007, pp. 327-30

[12] R.T. Howe, T.A. Roessig, A.P. Pisano, Nonlinear mixing in surface-micromachined tuning fork oscillators, in Frequency Control Symposium, 1997, Proc. IEEE Int., 1997, pp. $778-782$

[13] V. Kaajakari, J.K. Koskinen, T. Mattila, Phase noise in capacitively coupled micromechanical oscillators, IEEE Trans. Ultrason. Ferroelec. Freq. Contr. 52 (2005) 23222331

[14] C. Seoánez, F. Guinea, A.H. Castro Neto, Dissipation in graphene and nanotube resonators, Phys. Rev. B 76 (2007) 125427

[15] V. Sazonova, A tunable carbon nanotube resonator, Ph.D. Dissertation, Cornell University, Ithaca, New York, 2006

[16] L.D. Landau, E.M. Lifshitz, Theory of Elasticity, Butterworth-Heinemann, Oxford, 3rd edition, 1986

[17] H. Nishiyama, M. Nakamura, Capacitance of a strip capacitor, IEEE Trans. Compon. Hybrids Manuf. Technol. 13 (1990) 417-423

[18] A.H. Nayfeh, M.I. Younis, E.M. Abdel-Rahman, Dynamic pull-in phenomenon in mems resonators, Nonlinear Dyn. 48 (2007) 153-163

[19] C. Thouzé, O. Thomas, Non-linear behaviour of free-edge shallow spherical shells: Effect of the geometry, Int. J. Non-Linear Mech. 41 (2006) 678-692

[20] N. Kacem, Nonlinear dynamics of M\&NEMS resonant sensors: design strategies for performance enhancement, Ph.D. Dissertation, Insa-Lyon, CEA-LETI, Grenoble, 2010

[21] A. Husain, J. Hone, H.W. Ch. Postma, X.M.H. Huang, T. Drake, M. Barbic, A. Scherer, M.L. Roukes, Nanowirebased very-high-frequency electromechanical resonator, Appl. Phys. Lett. 83 (2003) 1240-1242

[22] A.H. Nayfeh, D.T. Mook, Nonlinear Oscillations, Wiley, 1979 
[23] C. Gui, R. Legtenberg, H.A.C. Tilmans, J.H.J. Fluitman, M. Elwenspoek, Nonlinearity and hysteresis of resonant strain gauges, in Micro Electro Mechanical Systems, 1995 , MEMS '95, Proc. IEEE, 1995, pp. 157-162

[24] L.C. Shao, M. Palaniapan, W.W. Tan, The nonlinearity cancellation phenomenon in micromechanical resonators, J. Micromech. Microeng. 18 (2008) 065014

[25] N. Kacem, S. Hentz, Bifurcation topology tuning of a mixed behavior in nonlinear micromechanical resonators, Appl. Phys. Lett. 95 (2009) 183104

[26] V. Kaajakari, T. Mattila, A. Lipsanen, A. Oja, Nonlinear mechanical effects in silicon longitudinal mode beam resonators, Sens. Actuators A: Physical 120 (2005) 64-70
[27] P.M. Osterberg, S.D. Senturia, M-test: A test chip for mems material property measurement using electrostatically actuated test structures, J. Microelectromech. Syst. 6 (1997) 107-118

[28] N. Kacem, S. Hentz, H. Fontaine, V. Nguyen, P. Robert, B. Legrand, L. Buchaillot, From mems to nems: Modelling and characterization of the non linear dynamics of resonators, a way to enhance the dynamic range, in Int. Conf. Nanotech, Boston, Massachusetts, USA, 2008

[29] H.M. Ouakad, M.I. Younis, Nonlinear dynamics of electrically-actuated carbon nanotube resonator, J. Comput. Nonlinear Dynam. 5 (2010) 011009 